\title{
CAMADA LIMITE NOTURNA SOBRE ÁREA DE PASTAGEM NA AMAZÔNIA
}

\author{
THEOMAR TRINDADE DE ARAÚJO TIBURTINO NEVES ${ }^{1}$, GILBERTO FISCH²
}

\author{
${ }^{1}$ Instituto Nacional de Pesquisas Espaciais/Centro de Previsão de Tempo e Estudos Climáticos (INPE/ \\ CPTEC), São José dos Campos, SP, Brasil \\ ${ }^{2}$ Instituto Tecnológico de Aeronaltica/Intituto de Aeronáutica e Espaço (ITA/IAE), São José dos Campos, SP, \\ Brasil
}

theomar.neves@cptec.inpe.br, gfisch@iae.cta.br

Recebido Junho de 2010 - Aceito Junho de 2011

\begin{abstract}
RESUMO
Neste trabalho foram discutidas as características da Camada Limite Noturna (CLN) e o regime turbulento predominante, durante a campanha DRY-to-WET, período de transição seco para chuvoso (setembro a novembro) do experimento RaCCI/LBA 2002. Foi utilizado um sítio de pastagem (Faz. Nossa Senhora Aparecida - $10^{\circ} 45^{\prime} \mathrm{S}, 62^{\circ} 21^{\prime} \mathrm{W}, 290 \mathrm{~m}$ ) na região de Ouro Preto D'Oeste - RO, com medidas de radiossonda (RS), balão cativo (BC), estação meteorológica automática (EMA) e de equipamento de sensoriamento remoto (SODAR). A determinação do regime turbulento ocorreu através do parâmetro de Monin-Obukhov. As alturas da CLN obtidas por várias técnicas (RS, BC e SODAR), que variaram de 156,3 $\pm 57,9 \mathrm{~m}$ ao pôr-do-sol a $301,0 \pm 124,5 \mathrm{~m}$ no início da manhã do dia seguinte, apresentaram semelhança com resultados já obtidos na estação seca da mesma região. Entretanto a característica da estabilidade atmosférica foi similar a situações obtidas na estação úmida. Ou seja, durante o período de transição entre as estações seca e chuvosa, a atmosfera não fica totalmente estabelecida apresentando características tanto da estação seca (por exemplo os valores da altura da CLN) como da úmida (características da estabilidade).
\end{abstract}

Palavras-chave: radiossondagem, balão cativo, SODAR, altura da camada limite noturna

\begin{abstract}
NOCTURNAL BOUNDARY LAYER OVER AN AMAZONIAN PASTURE AREA This paper discuss the characteristics of the nocturnal boundary layer (NBL) and the prevailing turbulent regime during the campaign DRY-to-WET, dry to wet season transition period (SeptemberNovember) of RaCCI/LBA 2002. Measurements of radiosonde (RS), tethered balloon (TB), automatic weather station (AWS) and SODAR (remote sensing device) were made over the pasture site (Faz. Nossa Senhora Aparecida - $10^{\circ} 45^{\prime} \mathrm{S}, 62^{\circ} 21^{\prime} \mathrm{W}, 290 \mathrm{~m}$ ) in the region of Ouro Preto D'Oeste - RO. The turbulent flow regime was determined using the Monin-Obukhov parameter. The NBL heights (RS, BC and SODAR), ranging from $156.3 \pm 57.9 \mathrm{~m}$ at sunset up to $301.0 \pm 124.5 \mathrm{~m}$ on the early morning of the next day, showed similarity with data already obtained during the dry season over the same region. However the characteristic of the atmospheric stability was similar to previous data collected during the wet season. In other words, during the transition period from dry to wet conditions, the atmosphere is not fully established, showing characteristics of both seasons: the height of the NBL is similar to the ones during dry period measurements while the atmospheric stability presents the same behavior as during the wet period. .
\end{abstract}

Keywords: nocturnal boundary layer, transition season, turbulent regime. 


\section{INTRODUÇÃO}

A Camada Limite Atmosférica (CLA) é a parte baixa da atmosfera que atua como ligação entre a superfície e a circulação de larga escala acima (atmosfera livre). A CLA efetua transportes verticais de energia, momentum e propriedades escalares que, por sua vez, agem modificando a dinâmica e a termodinâmica das circulações de larga escala por alterações na superfície e, de modo inverso, nos fluxos turbulentos de superfície (Fisch et al., 2004).

A CLA possui processos de geração de turbulência térmica e mecânica, sendo a produção ou destruição da turbulência térmica representada pelo empuxo e com a supressão do aquecimento da superfície produz-se o modo mecânico devido ao cisalhamento do vento. Esses processos são controlados pelas interações da superfície e do entranhamento com a atmosfera livre acima. Ao mesmo tempo, a CLA deve também modificar os fluxos de superfície através da influência da temperatura e umidade do ambiente, resultado da partição de energia da superfície (Fisch et al., 2004). A fase da CLA que ocorre em resposta ao resfriamento da superfície, tipicamente durante o período noturno e devido à emissão de radiação de onda longa para o espaço, é conhecida como Camada Limite Noturna (CLN). Como esta fase ocorre normalmente durante o período de estratificação estável, ela também é denominada de Camada Limite Estável (CLE).

Os processos atmosféricos que caracterizam a CLN são de escala de tempo da ordem de algumas horas, com extensão vertical máxima em torno de 500 m (Stull, 1988; Holtslag e Duynkerke, 1998), sendo característica marcante a presença de uma camada de inversão térmica próxima à superfície. Em condições de céu claro e de ventos com velocidades de moderados a fracos, a CLN é claramente definida (Tombrou et al., 1998), sendo que a turbulência é essencialmente gerada por cisalhamento do vento, a qual é altamente intermitente. Os empuxos negativos existentes devido ao fato do maior resfriamento da superfície em comparação com a atmosfera acima, agem para suprimir a turbulência mecânica e diminuir sua profundidade, assim como, os movimentos atmosféricos (tais como ondas de gravidade) podem coexistir com a turbulência, tornando sua estrutura mais complexa (Santos et al., 2007). Por outro lado, Santos et al. (2007) afirmam ainda que os efeitos radiativos de emissão de ondas longas da superfície atuam no desenvolvimento e aprofundamento da camada de inversão, que pode existir em condições de calmaria e, portanto, na ausência de turbulência.

Apesar do aumento de estudos da CLN para vários tipos de biomas, ainda há uma grande complexidade e pouco conhecimento dos parâmetros que descrevem sua estrutura, sobretudo em regiões como a Amazônia, onde a biosfera e atmosfera interagem de forma tão intensa e complexa. Outro papel importante da CLN é que o conhecimento de sua dinâmica tem sido pouco explorado no desenvolvimento de modelos atmosféricos de meso e grande escala, o que acaba por prejudicar a eficiência destes modelos em prognosticar o tempo e o clima daquela região (Santos, 2005a).

Segundo Fearnside (2006), o desmatamento na Amazônia brasileira tem aumentado continuamente desde 1991, variando de acordo com a situação econômica presente: economia bem aquecida provoca taxas altas de desmatamento, ao passo que períodos de recessão preservam a floresta tropical. Os dados de desmatamento, disponibilizados pelo Instituto Nacional de Pesquisas Espaciais (INPE), mostram que a estimativa da taxa de desflorestamento foi de $7.008 \mathrm{~km}^{2}$ para 2009, com uma margem de erro de 4\% (INPE, 2009). Para o desmatamento de larga escala, vários estudos numéricos (Oyama e Nobre, 2003; Costa e Yanagi, 2006; Godoy et al., 2009) postulam que poderá haver mudanças nas características atmosféricas. Contudo o impacto de meso-escala do desmatamento e seus efeitos na CLA ainda não são bem conhecidos (Fisch et al., 2004).

Este trabalho tem por objetivo aumentar o conhecimento científico da CLN sobre uma região de área desmatada (pastagem) na Amazônia, descrevendo seu desenvolvimento durante um período de transição da época seca para a chuvosa, por meio de diferentes tipos de dados coletados na campanha de campo RaCCI/LBA 2002. São determinadas suas características observacionais principais, bem como, se compara os resultados obtidos com pesquisas anteriores (Fisch, 1995; Santos, 2005a) para a mesma região. Adicionalmente, avalia a potencialidade da utilização de um instrumento de sensoriamento remoto (SODAR) para estudos da CLN.

\section{MATERIAIS E MÉTODOS}

O sítio é uma área desmatada (pastagem) no município de Ouro Preto do Oeste - RO, a sudoeste da Amazônia. As medidas foram realizadas na fazenda Nossa Senhora Aparecida $\left(10^{\circ} 45^{\prime} \mathrm{S}, 62^{\circ} 21^{\prime} \mathrm{W}, 290 \mathrm{MSL}\right)$ que é basicamente coberta por gramínea rasteira (Brachiaria brizantha) e é destinada para criação de gado. Os dados foram coletados durante o projeto RaCCI/LBA (Interação entre Radiação, Nuvens e Clima na Amazônia/ Large Scale Biosphere-Atmosphere Experiment in Amazonia) realizado no período de 06 de setembro a 05 de novembro de 2002, visando estudar características de transição da atmosfera entre as estações seca e chuvosa.

Durante o projeto $\mathrm{RaCCI} / \mathrm{LBA}$, foram feitas medições simultâneas dos principais elementos climáticos por meio de:

- Estação Meteorológica Automática (EMA) com medidas a cada minuto durante $24 \mathrm{~h}$, sendo que neste trabalho serão usadas as informações das 18 às 08 Hora Local (HL); 
- Perfilamento de Balão Cativo (BC) obtidos pelo Sistema de Aquisição de Dados Atmosférico (ADAS - "Atmospheric Data Acquisition System") da A. I. R. Inc. (EUA) que coletou informações sobre temperaturas do ar seco e úmido (com ventilação forçada), pressão atmosférica, velocidade e direção do vento, sendo realizados apenas 61 içamentos entre os dias 20 e 30 de outubro de 2002 (11 dias). Este pequeno período de dados foi devido a problemas de disponibilidade do equipamento para a campanha de campo;

- Lançamento de Radiossondagens (RS) com a sonda RS80-15G do modelo Digicora (MW12) da Vaisala (Finlândia), totalizando 142 lançamentos noturnos às 20, 02 e $08 \mathrm{HL}$, medindo dados de temperatura de ar, umidade relativa, pressão atmosférica e ventos (velocidade e direção);

- Sondagens remotas através de um SODAR (Sondador Acústico da Atmosfera) da marca REMTECH (França) com perfis médios armazenados continuamente no experimento a cada $30 \mathrm{~min}$. O instrumento é monoestático e funciona com uma potência de $10 \mathrm{~W}$ e freqüência aproximada de $2 \mathrm{kHz}$, sendo que a antena emissora/receptora tem uma área de $1,96 \mathrm{~m}^{2}$.

A determinação da altura da CLN, representada pelo nível onde termina um perfil estável, não é tão bem definida pelos perfis de temperatura potencial e umidade, como é o caso da Camada Limite Convectiva (CLC). Ainda assim, os métodos analíticos ainda são os mais confiáveis. Dessa maneira, visando avaliar a evolução temporal da CLN, foram utilizados os perfis de temperatura potencial $(\theta)$ organizados em perfis médios para cada horário, sendo as características médias da CLN também determinadas por estes perfis. Outra abordagem para o cálculo de altura da CLN foi à determinação da altura do vento máximo obtida pelo SODAR. Tal método de observação, sugerido por Stull (1988), foi utilizado devido ao fato que o algoritmo comercial, que está associado ao equipamento SODAR, identifica a Camada Residual e não a altura da CLN.

As características da CLN determinadas neste trabalho foram: altura da CLN (hi) - definida como a altura na qual o gradiente de temperatura potencial $(\theta)$ é nulo ou menor que 0,01 $\mathrm{K} \mathrm{m}^{-1}$ (valor determinado em função da precisão das medidas de temperatura da série de dados); $\theta$ (hi) como a temperatura no topo da camada (na altura hi); diferença entre $\theta$ (hi) e $\theta(\mathrm{s})$, onde o subscrito "s" significa superfície, determinando a descontinuidade térmica $(\Delta \theta)$; intensidade da inversão térmica (I), dada em $\mathrm{K} \mathrm{km}^{-1}$, é igual à $\Delta \theta$ dividida pela espessura da camada (hi); e a velocidade do vento (V) na altura da CLN (hi) em $\mathrm{m} \cdot \mathrm{s}^{-1}$. Visando as comparações dos resultados entre a transição das estações seca e chuvosa (ver Tabelas 1 e 2), utilizou-se os resultados provenientes de Fisch (1995), com o Experimento de Camada Limite de Rondônia - RBLE 2 e 3 - nas épocas secas de 1993 e 1994, e Santos (2005a), com o RBLE 3 e WetAMC-LBA (Campanha de Mesoescala Atmosférica da Estação Úmida do experimento LBA) ocorrido em 1999.

Para a determinação do regime turbulento utilizou-se

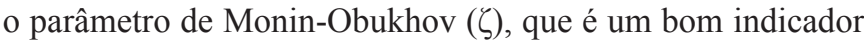
de estabilidade. $\mathrm{O}$ parâmetro de $\zeta$ é determinado a partir de variáveis de escala da camada limite superficial (Arya, 2001), a saber:

$$
\zeta=\frac{z}{L}=\frac{\left(g / T_{o}\right)\left(\overline{w^{\prime} \theta^{\prime}}\right)_{s}}{-u_{*}^{3} / t}
$$

Tabela 1 - Valores da altura da camada limite (hi), temperatura potencial virtual no topo da camada $(\theta v(h i))$ e descontinuidade da inversão térmica $(\Delta \theta \mathrm{v})$ em condições noturnas, para a pastagem durante a estação seca nos experimentos RBLE 2 (1993) e RBLE 3 (1994).

\begin{tabular}{c|c|c|c}
\hline \multicolumn{4}{c}{ RBLE 2 } \\
\hline $\mathrm{HL}$ & hi $(\mathrm{m})$ & $\theta \mathrm{v}_{\text {(hi) }}(\mathrm{K})$ & $\Delta \theta \mathrm{v}(\mathrm{K})$ \\
\hline $20: 00$ & 140 & 305,3 & 8,5 \\
\hline $23: 00$ & 150 & 303,9 & 9,2 \\
\hline $02: 00$ & 200 & 303,8 & 10,6 \\
\hline $05: 00$ & 240 & 303,9 & 11,6 \\
\hline- & - & - & - \\
\hline
\end{tabular}

\begin{tabular}{c|c|c|c}
\hline \multicolumn{4}{|c}{ RBLE 3 } \\
\hline HL & hi (m) & $\theta \mathrm{v}_{\text {(hi) }}(\mathrm{K})$ & $\Delta \theta \mathrm{v}(\mathrm{K})$ \\
\hline $18: 00$ & 70 & 306,7 & 2,6 \\
\hline $19: 00$ & 120 & 305,9 & 6,0 \\
\hline $21: 00$ & 160 & 305,3 & 7,9 \\
\hline $00: 00$ & 190 & 304,6 & 8,5 \\
\hline $05: 00$ & 230 & 304,1 & 10,5 \\
\hline
\end{tabular}

Fonte: Adaptado de Fisch (1995)

Tabela 2 - Valores da altura da camada limite (hi), temperatura potencial virtual no topo da camada ( $\theta$ (hi)), umidade específica (q) e da descontinuidade da inversão térmica $(\Delta \theta)$ e intensidade da inversão (I) em condições noturnas, para a pastagem durante a estação (a) seca (RBLE 3) e (b) úmida (WET AMC/LBA) da Amazônia.

\begin{tabular}{c|c|c|c|c|c}
\hline \multicolumn{7}{c}{ Estação seca (RBLE 3 1994) } \\
\hline HL & hi (m) & $\theta_{\text {(hi) }}(\mathrm{K})$ & $\mathrm{q}\left(\mathrm{g} \mathrm{kg}^{-1}\right)$ & $\Delta \theta(\mathrm{K})$ & $\mathrm{I}\left(\mathrm{K} \mathrm{km}^{-1}\right)$ \\
\hline $18: 00$ & 120 & 305,7 & 7,2 & 3,0 & 25,4 \\
\hline $19: 00$ & 110 & 304,5 & 7,3 & 5,6 & 50,5 \\
\hline $21: 00$ & 260 & 307,3 & 10,0 & 10,4 & 39,8 \\
\hline $00: 00$ & 230 & 303,3 & 9,0 & 9,1 & 39,4 \\
\hline $05: 00$ & 320 & 303,5 & 8,2 & 11,4 & 35,7 \\
\hline $07: 00$ & 320 & 303,3 & 8,2 & 10,4 & 32,4 \\
\hline
\end{tabular}

\begin{tabular}{c|c|c|c|c|c}
\hline \multicolumn{7}{c}{ Estação úmida (LBA 1999) } \\
\hline (b) & hi (m) & $\theta_{\text {(hi) }}(\mathrm{K})$ & $\mathrm{q}\left(\mathrm{g} \mathrm{kg}^{-1}\right)$ & $\Delta \theta(\mathrm{K})$ & $\mathrm{I}\left(\mathrm{K} \mathrm{km}^{-1}\right)$ \\
\hline $19: 00$ & 210 & 302,3 & 17,6 & 3,0 & 14,1 \\
\hline $21: 00$ & 207 & 300,4 & 17,7 & 2,6 & 12,6 \\
\hline $22: 00$ & 264 & 301,7 & 17,5 & 3,5 & 13,4 \\
\hline $01: 00$ & 227 & 301,0 & 17,7 & 3,0 & 13,2 \\
\hline $04: 00$ & 235 & 300,8 & 17,8 & 3,5 & 15,0 \\
\hline $07: 00$ & 156 & 299,9 & 17,9 & 0,6 & 4,0 \\
\hline
\end{tabular}

Fonte: Adaptado de Santos (2005a). 
onde $u_{*}$ é a velocidade de fricção $\left[\mathrm{m} \mathrm{s}^{-1}\right]$, $\mathrm{k}$ a constante de Von Karman $(\approx 0,4),\left(\mathrm{g} / \mathrm{T}_{0}\right)$ o parâmetro de flutuabilidade $\left[\mathrm{m} \mathrm{s}^{-2}\right.$ $\left.\mathrm{K}^{-1}\right]$ e $\left(\overline{w^{\prime} \theta^{\prime}}\right)_{s}$ o fluxo de calor à superfície $\left[\mathrm{W} \mathrm{m}^{-2}\right]$. Estes foram calculados para intervalos de tempo de $30 \mathrm{~min}$. Os critérios de estabilidade atmosférica são baseados no sinal de $\zeta$, a sabe: $\zeta$ $>0$ indica estabilidade atmosférica; $\zeta=0$ indica condição de neutralidade e $\zeta<0$ indica atmosfera instável. Foi adotada a metodologia proposta por Mahrt et al. (1998) para caracterizar a intensidade da estabilidade da seguinte maneira:

a) Regime pouco estável (RPE) - $0<\mathrm{z} / \mathrm{L} \leq \varepsilon 1$;

b) Regime de transição (RT) $-\varepsilon 1<\mathrm{z} / \mathrm{L} \leq \varepsilon 2$;

c) Regime muito estável (RME) - z/L $>\varepsilon 2$;

A constante $\varepsilon$ representa o valor de $\zeta$, no qual se observa um máximo valor de fluxo de calor negativo. Santos (2005a) utilizou esta mesma metodologia para a região da pastagem na Amazônia (dados do WetAMC) e sugere valores de $\varepsilon 1=10,8$ e $\varepsilon 2=11,8$.

Segundo Mahrt et al. (1998), para estratificação muito fraca, a magnitude do fluxo de calor para baixo é limitada pela flutuação da temperatura e o fluxo de calor desaparece quando estratificação da temperatura também desaparece (estratificação neutra). Já com a forte estratificação o fluxo de calor para baixo se torna limitado pelas restrições da flutuação dos movimentos verticais. E entre alguns valores da estabilidade entre dois regimes limitados, o fluxo de calor para baixo atinge um máximo. O que torna esse método eficaz na análise do regime.

\section{RESULTADOS}

a) Características dos elementos de superficie durante época de transição

As condições de superfície (Figura 1a) no período noturno (das 18 às $8 \mathrm{HL}$ ) apresentaram uma temperatura do ar com padrões normais, com um valor médio de $23,2 \pm 0,9^{\circ} \mathrm{C}$, ocorrendo o valor mínimo às $05 \mathrm{HL}\left(21,7 \pm 0,5^{\circ} \mathrm{C}\right)$. A evolução da temperatura mostrou um resfriamento de $-0,3{ }^{\circ} \mathrm{C} \mathrm{h}^{-1}$ a partir das $18 \mathrm{HL}$, cessando no nascer do Sol (às $6 \mathrm{HL}$ ). A umidade específica apresentou uma lenta diminuição durante à noite, sendo o valor médio encontrado de $16,9 \pm 0,4 \mathrm{~g} \mathrm{~kg}^{-1}$. O valor máximo foi de 17,4 $\pm 0,3 \mathrm{~g} \mathrm{~kg}^{-1}$ ocorrendo desde às 18 até às $21 \mathrm{HL}$. O vento em superfície foi fraco, com velocidade média ao redor de 1,0 $\mathrm{m} \mathrm{s}^{-1}$, sendo a predominância do vento de Norte (dados não apresentados).

Analisando ainda a Figura 1a, o saldo de radiação (Rn) torna-se negativo a partir das $18 \mathrm{HL}$ com valor típico da ordem de $-25,0 \mathrm{~W} \mathrm{~m}^{-2}$, retornando para valores positivos por volta das $06 \mathrm{HL}$, devido ao início do aquecimento solar. O mínimo do saldo de radiação ocorreu às $18 \mathrm{HL}$ com $-42,5 \pm 3,7 \mathrm{~W} \mathrm{~m}^{-2}$, crescendo até às $21 \mathrm{HL}\left(-31,9 \pm 2,7 \mathrm{~W} \mathrm{~m}^{-2}\right)$ e estabilizando-se no restante da noite em torno de $-30 \mathrm{~W} \mathrm{~m}^{-2}$. Estes valores são semelhantes aos já observados anteriormente, neste mesmo local em outra época do ano (Von Randow et al., 2004). Durante todo o experimento foi observada uma quantidade total de chuva de $227,5 \mathrm{~mm}$, porém ocorreu apenas $17,1 \%(24,0 \mathrm{~mm})$ da precipitação no período noturno: a maior concentração foi às 07 HL $(7,7 \%$ dos eventos equivalente a $10,4 \mathrm{~mm})$. Comparando as características das estações seca e úmida (Figura 1b e 1c) percebe-se apenas uma diferença na velocidade do vento entre 00 a $02 \mathrm{HL}$, que mostra um máximo de alta velocidade no período úmido, devido a precipitação umedecer o solo, diminuindo a friç̧ão e aumentando a magnitude do vento.

\section{b) Características básicas da CLN}

Inicialmente, determinou-se as características médias horárias da CLN através dos içamentos do BC (Tabela 3), que compreenderam um período pequeno de 11 dias (de 20 a 30 de Outubro de 2002) do experimento. Esta pequena amostra deu-se devido a disponibilidade do equipamento somente na parte final do experimento LBA/RACCI 2002. Os horários que tiverem menos de 4 içamentos não foram consideradas nesta tabela. É fato de que o BC é uma das melhores maneiras de se estimar as propriedades da CLN, porém o equipamento esteve disponível por poucos dias apenas. Dessa forma, optou-se também por comparar as informações obtidas pelo $\mathrm{BC}$ com outros métodos (RS e SODAR) e utilizar estes últimos para caracterizar a CLN durante o experimento LBA/RACC 2002. Observou-se que a CLN inicia com uma média de 95,2 $\pm 32,2$ m (às 18 HL), aumentando sua espessura até um valor de 247,2 $\pm 78,3 \mathrm{~m}$ no início da manhã seguinte (às $8 \mathrm{HL}$ ). A maior altura observada alcançou $329,0 \mathrm{~m}$, ocorrendo no dia $27 / 10$, às 8 HL. Neste dia foram observadas poucas células convectivas, sendo considerado um dia de céu limpo e, por conseguinte, de desenvolvimento clássico. Acevedo et al. (2004) observaram alturas de CLN menores do que os obtidos aqui, também em um sítio de pastagem na Amazônia (em Santarém-PA). Esta diferença de altura ocorre devido as diferentes condições geográficas, a medida que o sítio em Santarém se encontra próximo do encontro das águas dos Rios Tapajós e Amazônas, diferente do caso avaliado aqui, situado em Ouro Preto D'Oeste. Um exemplo da influência do fator geográfico é o fato de, no caso de Santarém, os autores terem observado vários casos de formação de nevoeiros durante a noite (sendo inclusive um critério para a determinação da CLN), ao passo que este evento meteorológico não ocorreu em Rondônia. Outro fator observado na distinção foi que no sítio de Ouro Preto D'Oeste, o vento era da ordem de $1 \mathrm{~m} \mathrm{~s}^{-1}$ superior ao observado em Santarém.

As temperaturas observadas no topo da camada $\left(\theta_{(\text {hi })}\right)$, ainda na Tabela 3, mostraram um maior aquecimento no início do período noturno e no final, sendo o maior às $19 \mathrm{HL} \operatorname{com} 302,3$ K. Já a menor temperatura foi encontrada antes do nascer do Sol, às $05 \mathrm{HL}(297,5 \mathrm{~K})$, obtida a partir de sete içamentos. A inversão térmica foi mais forte às $00 \mathrm{HL}\left(\operatorname{com} 34,8 \mathrm{~K} \mathrm{~km}^{-1}\right)$, indicando 


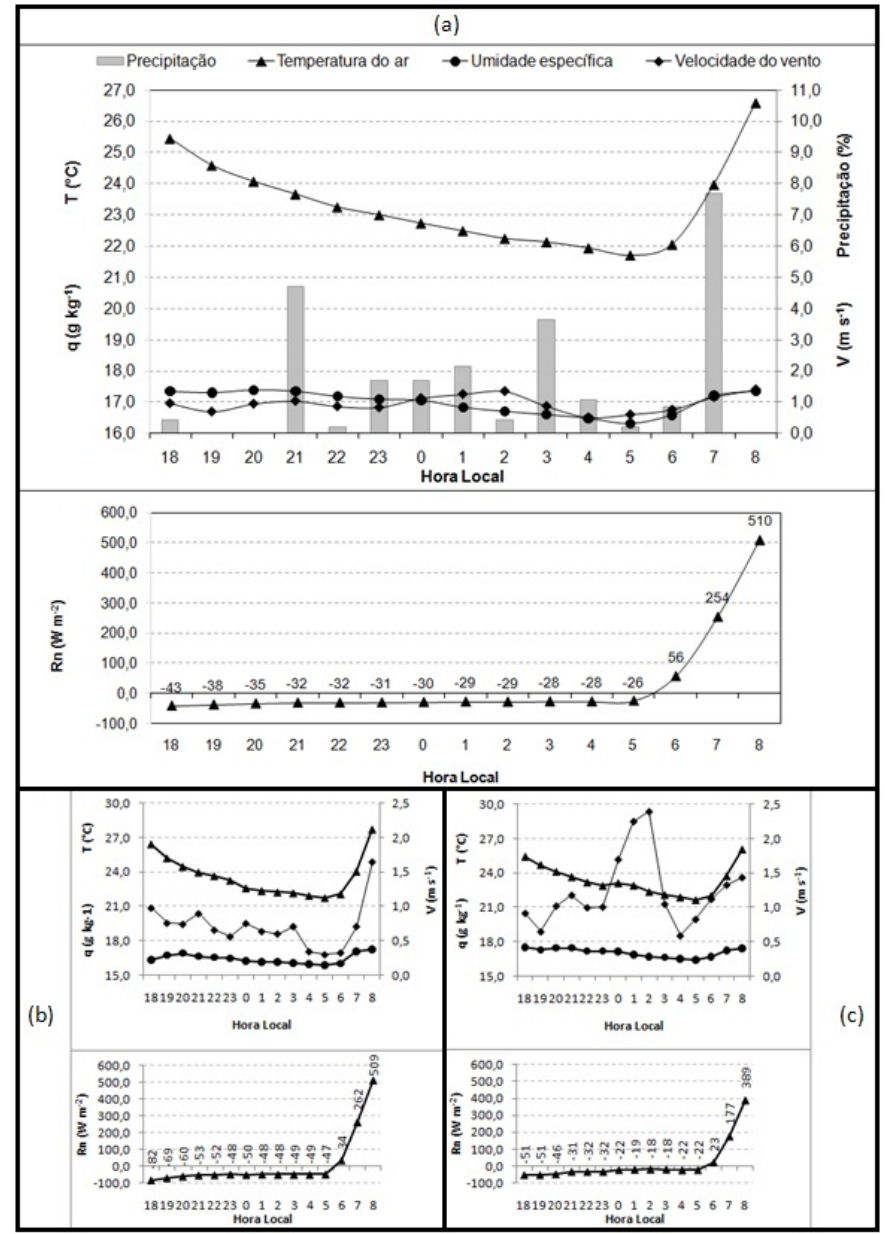

Figura 1 - Ciclos noturnos médios de superfície da (superior) temperatura do ar (T), umidade específica (q), velocidade do vento (V), precipitação (\%) e (inferior) saldo de radiação (Rn): (a) todo o experimento, dias representativo seco (b) e úmido (c).

uma grande estabilidade da CLN, pois foi praticamente o dobro do valor médio de todo o período $\left(20,5 \mathrm{~K} \mathrm{~km}^{-1}\right)$.

As taxas de aquecimento/resfriamento da superfície apresentaram um aquecimento após às $05 \mathrm{HL}$, devido ao nascer do Sol, sendo que a taxa máxima de aquecimento ocorreu às $07 \mathrm{HL}$ (aproximadamente 3,3 $\mathrm{K} \mathrm{h}^{-1}$ ). No geral, houve uma tendência de resfriamento no período noturno e de um fraco aquecimento após o nascer do Sol. A velocidade do vento variou entre $1,7 \mathrm{~m} \mathrm{~s}^{-1}$ (02 HL) e $4,8 \mathrm{~m} \mathrm{~s}^{-1}(00 \mathrm{HL})$, mostrando a presença de intermitência do vento (e turbulência). As maiores velocidades do vento ocorreram na primeira metade da noite (18 a $00 \mathrm{HL}$ ).

Os instrumentos de RS e SODAR foram utilizados para ressaltar as características de todo o período do experimento (de 06 de setembro a 05 de Novembro de 2002), devido a sua maior disponibilidade de dados. A radiossonda, apesar de um ser um instrumento muito útil no estudo da CLN, até mesmo devido ao maior número de lançamento em comparação com outros equipamentos (por exemplo balão cativo e/ou pipas), não é o instrumento ideal para análises da CLN devido a taxa de ascensão do balão ser muito rápida e a espessura da camada ser aproximadamente de 200 a $300 \mathrm{~m}$. Na Tabela 4, as alturas da CLN durante todo o experimento apresentaram um desenvolvimento regular, com uma taxa de crescimento de aproximadamente $12,4 \mathrm{~m} \mathrm{~h}^{-1}$ das 20 às $02 \mathrm{HL}$ (com uma variação total de $74,3 \mathrm{~m}$ ) e uma maior taxa de $15,9 \mathrm{~m} \mathrm{~h}^{-1}$ entre às $02 \mathrm{HL}$ e $08 \mathrm{HL}$ (variação total de 95,2 m). Ao analisar as características da termodinâmica da atmosfera, o que pôde ser constatado é que, na altura do topo da CLN, a tendência temporal de temperatura, umidade específica e inversão térmica é de diminuição ao longo da noite. As taxas de temperatura e umidade para as primeiras seis horas (20 às $02 \mathrm{HL}$ ) foram de cerca de $-0,2^{\circ} \mathrm{C} \mathrm{h}^{-1}$ e $-0,1 \mathrm{~g} \mathrm{~kg}^{-1} \mathrm{~h}^{-1}$, e para as seis horas posteriores ( 02 às $08 \mathrm{HL}$ ) estes valores são $-0,0^{\circ} \mathrm{C} \mathrm{h}^{-1} \mathrm{e}-0,1$ $\mathrm{g} \mathrm{kg}^{-1} \mathrm{~h}^{-1}$, respectivamente. Já a inversão térmica apresentou apenas uma grande diminuição nas últimas seis horas com $-1,8$ $\mathrm{K} \mathrm{km}^{-1} \mathrm{~h}^{-1}$. Esta diminuição da inversão térmica é devido ao efeito do nascer do Sol (por volta das $06 \mathrm{HL}$ ), que aquece a superfície e reduz a diferença de temperatura entre a superfície e um determinado nível. A curva horária da temperatura de superfície foi muito regular, não variando mais que $2,2 \mathrm{~K}$. O vento teve suas máximas velocidades às $02 \mathrm{HL}$, com uma média de $4,8 \mathrm{~m} \mathrm{~s}^{-1}$, seguido por máximo secundário de $4,5 \mathrm{~m} \mathrm{~s}^{-1}$ às $08 \mathrm{HL}$ e sendo registrada a ocorrência do vento mais forte na altura da CLN $\left(9,8 \mathrm{~m} \mathrm{~s}^{-1}\right)$. A frequência da direção do vento às $20 \mathrm{HL}$ foi dividida entre norte e noroeste $(27,8 \%)$, com um padrão de noroeste às $02 \mathrm{HL}(40,9 \%)$ e norte às $08 \mathrm{HL}(34,4 \%)$. Estes valores, apesar de superiores aos apresentados na Tabela 3 (oriundos da determinação do BC), mostram (qualitativamente) o mesmo comportamento da Tabela 3.

Tabela 3 - Valores médios da altura da CLN (hi), temperatura potencial no topo da CLN ( $\theta($ hi)), umidade específica (q), descontinuidade térmica $(\Delta \theta)$, intensidade da inversão térmica $(\mathrm{I})$, temperatura potencial na superfície $(\theta \mathrm{s})$ e velocidade do vento (V). Dados do balão cativo medidos entre 20 e 30 de outubro de 2002 .

\begin{tabular}{c|c|c|c|c|c|c|c|c}
\hline $\begin{array}{c}\text { Hora } \\
\text { Local }\end{array}$ & $\begin{array}{c}\mathrm{N}^{\mathrm{o}} \\
\text { perfis }\end{array}$ & hi (m) & $\theta_{(\mathrm{hi})}(\mathrm{K})$ & $\mathrm{q}\left(\mathrm{g} \mathrm{kg}^{-1}\right)$ & $\Delta \theta(\mathrm{K})$ & $\mathrm{I}\left(\mathrm{K} \mathrm{km}^{-1}\right)$ & $\theta \mathrm{s}(\mathrm{K})$ & ${\mathrm{V}\left(\mathrm{m} \mathrm{s}^{-1}\right)}$ \\
\hline $18: 00$ & 5 & $95,2 \pm 32,2$ & 301,6 & 16,2 & 1,8 & 19,9 & 299,8 & 2,6 \\
\hline $19: 00$ & 4 & $114,3 \pm 18,5$ & 302,3 & 16,5 & 2,8 & 25,2 & 299,5 & 4,8 \\
\hline $5: 00$ & 7 & $176,5 \pm 38,4$ & 297,5 & 15,3 & 3,3 & 18,5 & 294,3 & 2,7 \\
\hline $7: 00$ & 5 & $217,4 \pm 60,7$ & 300,2 & 15,9 & 2,6 & 11,0 & 297,6 & 3,6 \\
\hline $8: 00$ & 8 & $247,2 \pm 78,3$ & 300,9 & 16,3 & 0,7 & 3,0 & 300,1 & 3,3 \\
\hline
\end{tabular}


A termodinâmica da atmosfera foi analisada também através do cálculo dos valores de Energia Potencial Disponível para Convecção (CAPE $\left[\mathrm{J} \mathrm{kg}^{-1}\right]$ ) e Energia de Inibição da Convecção (CIN [ $\left.\mathrm{J} \mathrm{kg}^{-1}\right]$ ). Observou-se um valor médio de CAPE de $136,9 \pm 196,0 \mathrm{~J} \mathrm{~kg}^{-1}$, ao passo que o valor médio da CIN foi de 93,3 $\pm 104,0 \mathrm{~J} \mathrm{~kg}^{-1}$. Fisch et al. (2004) observaram que na estação úmida a condição diurna propicia um melhor desenvolvimento da convecção, valores de CAPE da ordem de $1000,0 \mathrm{~J} \mathrm{~kg}^{-1}$. Por outro lado, tanto para os períodos diurno, como no noturno, Mota e Nobre (2006) encontraram uma forte inibição da convecção (CIN) com média de $300 \mathrm{~J} \mathrm{~kg}^{-1}$ durante a época úmida da região de Rondônia. Fisch et al. (2004) calculando também o CAPE para a estação seca, verificou quase que cinco vezes menos energia disponível se comparada com a época úmida, mas ainda superior (em 43,1 $\mathrm{J} \mathrm{kg}^{-1}$ ) em relação a época de transição. Isto sugere uma pequena disponibilidade de energia de convecção no período noturno. As precipitações que ocorreram foram, provavelmente, resíduos de formações convectivas que se desenvolveram durante o dia. Durante o período diurno e para o mesmo sítio de medida deste estudo (pastagem na Amazônia), Santos (2005b) obteve um valor de CAPE da ordem de $2000 \mathrm{~J} \mathrm{~kg}^{-1}$.

c) Estimativas da altura da CLN usando o instrumento SODAR

Uma vez determinada as alturas da CLN com os instrumentos BC e RS, decidiu-se avaliar o uso do equipamento SODAR, que calcula a altura da inversão térmica através do software da fabricante REMTECH (Figura 2). O equipamento tem a vantagem de disponibilizar uma grande quantidade de dados verticais (perfis médios a cada $30 \mathrm{~min}$.), detalhando melhor as características da atmosfera e, por conseguinte, da CLN. Entretanto, observa-se claramente que os valores apresentados (baseados na altura da inversão térmica entre a transição da Camada Residual e a atmosfera livre) são muito superiores aos determinados nas Tabelas 3 e 4 . Este fato levou a implementar um outro tipo de cálculo para hi, baseado na altura do vento máximo. A altura média da CLN estimada por este novo método, obtida entre os horários das 18 às $8 \mathrm{HL}$, ficou em torno de $296,7 \pm 114,0 \mathrm{~m}$, enquanto para a altura da inversão térmica (critério utilizado pelo software REMTECH) apresentou um valor médio de $867,7 \pm 283,9 \mathrm{~m}$. A altura média obtida com o $\mathrm{BC}$ foi de $166,2 \pm 68,7 \mathrm{~m}$, mostrando que, apesar da superestimativa, o método do nível do vento máximo assemelha-se mais a realidade. A Figura 2 apresenta os valores médios horários para o período de 20 a 30 de Outubro, coincidente com as medidas de BC. Uma deficiência observada, tanto para o método REMTCH, como para o do nível de vento máximo, foi a de que o SODAR não apresenta uma transição entre as determinações das alturas da CLC e da CLN: no horário de transição do pôr-do-sol, a CLN deveria iniciar com uma altura zero e crescer com o resfriamento radiativo da superfície. Além disso, nota-se pouca ou praticamente nenhuma variação temporal. Os dados obtidos pelo BC mostram claramente o aprofundamento da CLN com a evolução temporal noturna.

Na Figura 3 são apresentados os valores médios das alturas da CLN para todo o período do experimento (de 06 de Setembro a 05 de Novembro de 2002), das 18 às $08 \mathrm{HL}$, e estimando-se seu início por volta das $17 \mathrm{HL}$. Oliveira e Fisch (2000) determinaram que o horário de inversão do fluxo de calor sensível em áreas de pastagem na Amazônia é por volta de 16:43 HL, sendo que neste experimento (RaCCI/LBA 2002), esta inversão se deu às 17:34 HL. A diferença de tempo deve-se à época do ano utilizada por Oliveira e Fisch (verão) e este trabalho (primavera). Assim, assumiu-se que as $17 \mathrm{HL}$ a CLN possuía uma altura nula, desenvolvendo-se para 201,7 $\pm 60,5 \mathrm{~m}$ às $18 \mathrm{HL}$. Os valores médios horários apresentam alturas entre 214,8 e 289,6 m durante toda a evolução noturna. O comportamento da altura, no geral, para o SODAR foi de um crescimento lento e contínuo (em torno de $6,0 \mathrm{~m} \mathrm{~h}^{-1}$ ). Entretanto, a variabilidade (estimada pelos desvios padrões) é alta, mostrando a ocorrência de intermitência da turbulência. $\mathrm{O}$ valor médio da altura da CLN para todo o período noturno foi de $263,5 \pm 121,7 \mathrm{~m}$. Este valor está mais próximo aos observados pelo BC, bem como daqueles estimados pela RS (embora esta última seja superior).

Com um objetivo de detalhar mais as informações obtidas com o SODAR, o conjunto de dados foi dividido em duas fases, a saber: fase I - período mais seco (11 de setembro a 07 de outubro) e fase II - período mais chuvoso ( 08 de outubro a 03 de novembro), da época de transição. Estes mesmos períodos foram utilizados por Santos (2005b) para análise da CLC. Na Figura 4 as alturas da CLN mostraram que na fase I o desenvolvimento atingiu uma média de 258,3 m (com um máximo de 337,5 m), enquanto na época mais úmida (fase II) atingiu a altura de 267,2

Tabela 4 - Valores médios da altura da CLN (hi), temperatura potencial no topo da CLN ( $\theta($ hi)), umidade específica (q), descontinuidade térmica $(\Delta \theta)$, intensidade da inversão térmica (I), temperatura potencial na superfície $(\theta$ s), velocidade $(\mathrm{V})$ e direção (D) do vento, obtidos com a radiossonda em todo experimento RaCCI/LBA 2002.

\begin{tabular}{c|c|c|c|c|c|c|c|c|c}
\hline $\mathrm{HL}$ & $\begin{array}{c}\mathrm{n}^{\mathbf{0}} \\
\text { perfis }\end{array}$ & $\mathrm{h}_{\mathrm{i}}(\mathrm{m})$ & $\theta_{\text {(hi) }}(\mathrm{k})$ & $\mathrm{q}\left(\mathrm{g} \mathrm{kg}^{-1}\right)$ & $\Delta \theta(\mathrm{K})$ & $\mathrm{K} \mathrm{km}^{-1}$ & $\theta \mathrm{s}(\mathrm{K})$ & ${\mathrm{V}\left(\mathrm{m} \mathrm{s}^{-1}\right)}$ & $\mathrm{D}\left({ }^{\circ}\right)$ \\
\hline 20 & 36 & $172,1 \pm 81,0$ & 304,0 & 13,8 & 3,6 & 22,4 & 300,3 & 3,9 & $\mathrm{~N}-\mathrm{NO}$ \\
\hline 02 & 22 & $246,4 \pm 89,1$ & 302,8 & 13,4 & 4,7 & 20,0 & 298,1 & 4,8 & $\mathrm{NO}$ \\
\hline 08 & 32 & $341,6 \pm 87,0$ & 302,7 & 12,7 & 3,1 & 9,1 & 299,6 & 4,5 & $\mathrm{~N}$ \\
\hline
\end{tabular}




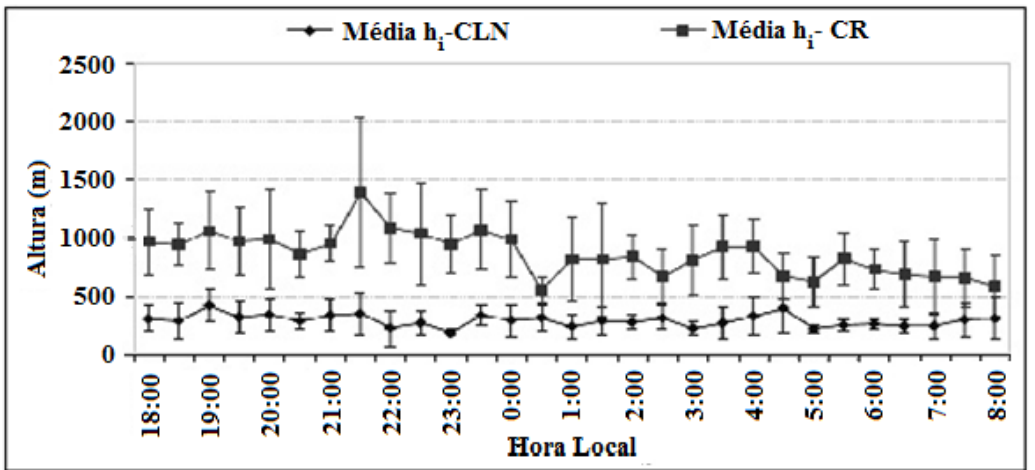

Figura 2 - Média horária das alturas da CLN e da CR obtidas com o SODAR no período de içamento do BC (20-30/10), experimento RaCCI/LBA.

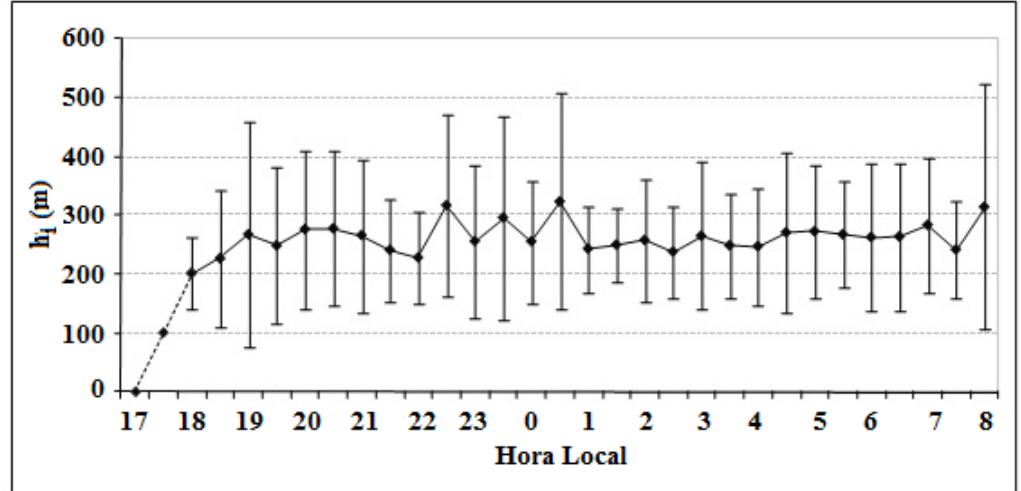

Figura 3 - Média horária das alturas da CLN com o SODAR para todo o período do experimento (06/09 à 05/11), sendo estimado o início da CLN a partir das 17 HL com a linha tracejada.

m (máximo 330,6 m). Quanto menos for aquecida a superfície durante o dia, mais facilmente se forma a CLN, por isso a fase II apresenta-se com maior desenvolvimento. Com o objetivo de estudar o crescimento temporal da CLN, ajustou-se uma equação do tipo (Stull, 1988):

$$
h_{i}=a \sqrt{t}
$$

sendo $h_{i}(m)$ a altura da CLN, a $\left(\mathrm{m} \mathrm{h}^{-1 / 2}\right)$ uma constante e $t(h)$ a variável que representa o tempo. As constantes para ambas as fases I e II são próximas (50,3 e 52,2 $\mathrm{m} \mathrm{h}^{-1 / 2}$, respectivamente), e mostram um coeficiente maior quando as condições são mais estáveis e propícias para maiores alturas da CLN.

Como as principais características da CLN foram medidas por diferentes equipamentos (BC, RS e SODAR), foi possível investigar o comportamento dos mesmos na determinação das características da CLN. As diferenças de medidas entre cada instrumento estão apresentadas na Tabela 5. Notou-se uma grande variação entre as diversas estimativas de alturas da CLN, sendo que a menor diferença foi obtida entre as alturas estimadas pelo BC e pela RS para 11 dias de dados $(-112,5 \pm 92,9 \mathrm{~m})$, devido a utilização do $\mathrm{BC}$ apenas compreender esse período de 11 dias, e a maior entre os dados oriundos da RS e do SODAR para todo o período de coleta de dados $(-175,7 \pm 159,3 \mathrm{~m})$. O sinal negativo indica subestimativa do primeiro instrumento sobre o segundo, indicando que o SODAR superestimou tanto o BC, quanto a RS. Analisando-se comparativamente os dados entre o BC e a RS, apenas a umidade específica $\left(4,7 \pm 2,4 \mathrm{~g} \mathrm{~kg}^{-1}\right)$ foi superestimada pelo $\mathrm{BC}$, enquanto as demais características foram subestimadas. Ressalta-se que a umidade específica é calculada por psicrometria para o instrumento $\mathrm{BC}$ e pelo sensor higrômetro para o RS. Para as características do vento entre o BC e a RS, as médias das diferenças foram menores, da ordem de $-0,9 \pm 1,4 \mathrm{~m} \mathrm{~s}^{-1} \mathrm{e}-58 \pm 233^{\circ}$, se comparadas com os resultados do SODAR. Vale salientar que o SODAR superestimou todos os parâmetros obtidos pelo BC e RS.

Através dos desvios padrões (Tabela 5) observa-se uma grande variabilidade entre as medidas, estando relacionados aos diferentes métodos de medidas utilizados por cada instrumento. Estas medidas são feitas de maneira direta, mas com sensores diferentes para o BC e a RS e indireta, para o SODAR.

Comparando as alturas da CLN dos 3 instrumentos com os resultados obtidos por Fisch (1995) e Santos (2005a), apresentadas nas Tabelas 1 e 2, nota-se uma maior semelhança dos dados deste experimento (RACCI/LBA) com a estação seca (RBLE 3) observada por Santos (2005a), onde a média da hi difere em apenas $2,0 \mathrm{~m}$. Isto sugere que a CLN no período de transição da época seca para a úmida, ainda possui grande influência da estação seca, ocasionando alturas mais altas no 


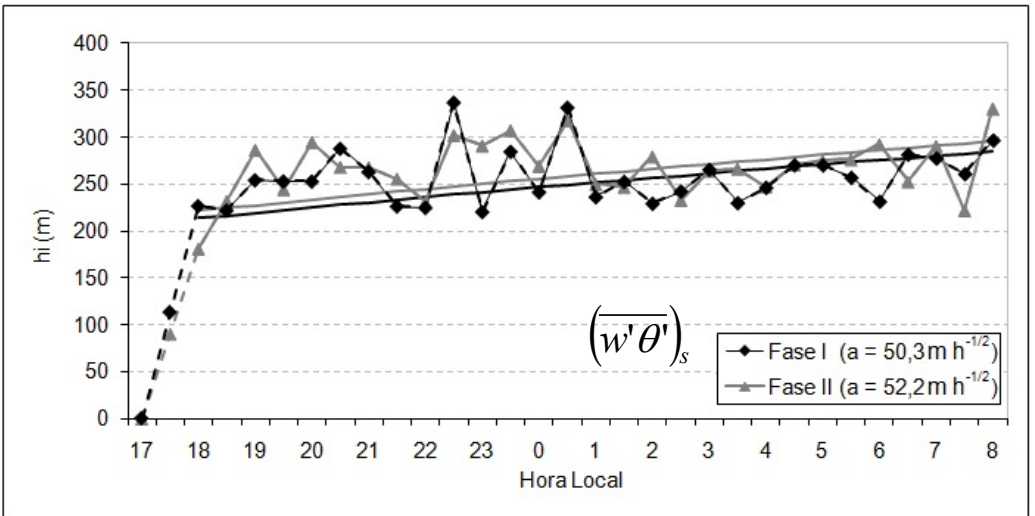

Figura 4 - Média horária das alturas da CLN com o SODAR, durante período de transição, estimando-se o inicio da CLN a partir das 17 HL (linha tracejada). Incluso o coeficiente de crescimento "a" (m h-1/2) obtido pela relação h $\mathrm{x} \sqrt{t}$.

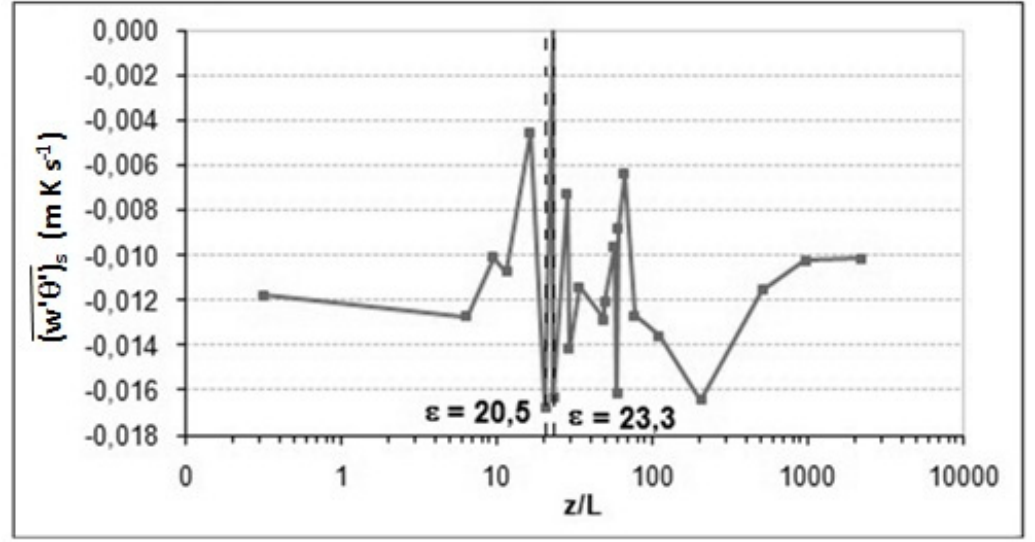

Figura 5 - Variação do fluxo de calor $\left(\left(\overline{w^{\prime} \theta^{\prime}}\right)_{s}\right)$ com a estabilidade $(\mathrm{z} / \mathrm{L})$.

início do experimento e mais baixas ao final. Entretanto, a inversão térmica (I) determina um padrão similar com a época úmida também observada por Santos (2005a). Ou seja, apesar das alturas serem mais similares à época seca, a inversão térmica é mais característica da época úmida, demonstrando que a atmosfera não está totalmente estabelecida, o que é característico de uma época de transição.

\section{d) Regime turbulento}

As características da turbulência atmosférica foram estudadas através do parâmetro de Monin-Obukhov (z/L), que possibilitou caracterizar os tipos de turbulência como proposto por Mahrt et al. (1998). Um estudo feito por Sakai et al (2004), com superfície semelhante ao localizado em Ouro Preto D'Oeste, relatou uma forte estabilidade o que tornaria $\mathrm{z} / \mathrm{L}$ inadequado, entretanto o mesmo não ocorre no sítio de Ouro Preto do D'Oeste devido a características como a velocidade de friç̧ão e geográficas que são distintas, pelo sítio em Santarém se encontrar próximo a confluência do Rio Tapajós e Amazonas. Assim, o uso de z/L é adequado na região. A definição dos limites numéricos para os regimes pouco e muito estáveis $(\varepsilon 1$ e ع2) foi dada através da organização de 23 medidas dos fluxos médios negativos de calor sensível (em K m s${ }^{-1}$ ) e confrontados com os valores médios do parâmetro de Monin-Obukhov. Os valores de $\varepsilon_{1}$ e $\varepsilon_{2}$ foram definidos como os valores de $\mathrm{z} / \mathrm{L}$ no primeiro $\left(0,017 \mathrm{~K} \mathrm{~m} \mathrm{~s}^{-1}\right)$ e no segundo máximo $(0,016 \mathrm{~K} \mathrm{~m}$ $\mathrm{s}^{-1}$ ) observados no fluxo negativo de calor, sendo os valores numéricos de $\varepsilon 1=20,5$ e $\varepsilon 2=23,3$, respectivamente (Figura 5). Os valores dos coeficientes determinados por Santos (2005a), para o mês de fevereiro de $1999(\mathrm{LBA} / \mathrm{TRMM})$ representando a estação chuvosa, foram de $\varepsilon 1=10,8$ e $\varepsilon 2=11,8$. Estes mesmos coeficientes ainda não haviam sido determinadas para o período seco desta região.

Durante a estação úmida, Santos (2005a) constatou uma CLN predominantemente sob o regime de turbulência fracamente estável. O período de transição também apresentou uma maior influência de regime pouco estável (RPE), apesar da ocorrência de alguns pontos com regime de muita estabilidade (RME), principalmente entre às 19 e $00 \mathrm{HL}$, além dos regimes de instabilidade ocorridos às 17 e 06:30 HL (Figura 6). Já se é sabido que a presença de intermitência é comum em condições estáveis (Holtslag e Nieuwstadt, 1986; Santos, 2005a), nesse estudo, a variação ocorreu devido à presença de intensas atividades convectivas, como alguns sistemas convectivos de mesoescala, que costumam aumentar os valores de umidade 


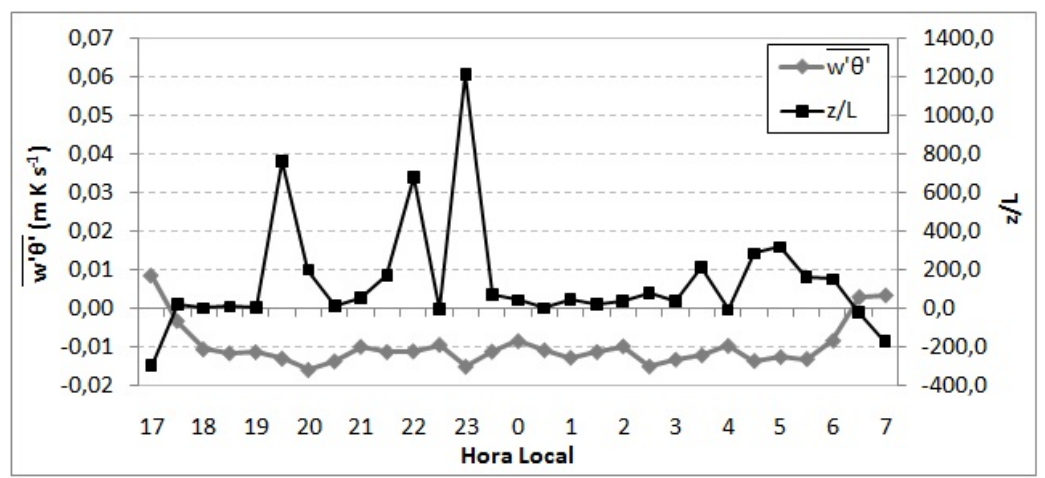

Figura 6 -Média horária do fluxo de calor e do parâmetro de estabilidade.

específica, principalmente na superfície, contribuindo assim com as variações de fluxo de calor na superfície (Figura 6). Os ventos observados para uma altura de $50 \mathrm{~m}$ (obtidos pelo equipamento SODAR) são da ordem de $2 \mathrm{~m} \mathrm{~s}^{-1}$ mostrando a existência da geração da turbulência pelo cisalhamento do vento.

\section{CONCLUSÃO}

O presente estudo foi realizado com o objetivo principal de analisar o desenvolvimento da CLN sobre uma região de pastagem na Amazônia, durante um período de transição da época seca para a chuvosa, durante a campanha RaCCI/LBA 2002. Como objetivos secundários avaliaram-se a utilização de um instrumento de sensoriamento remoto (SODAR) para estudos da CLN, bem como se caracterizou o regime turbulento predominante. O desenvolvimento da CLN foi determinado através de suas características observacionais e através de comparação com resultados de pesquisas anteriores nos períodos seco e úmido da mesma região.

Observou-se que, a partir de um conjunto de 11 dias de içamento com o balão cativo (BC), a altura da CLN apresentou um crescimento mais semelhante a estação seca. Durante as análises do período completo do experimento (de 06/09 a 05/11), verificou-se que o período de transição não possui uma atmosfera ainda totalmente estabelecida, pois tanto a RS, quanto o SODAR, apresentaram condições de crescimento semelhantes à época seca, enquanto a inversão térmica demonstrou uma estabilidade parecida com a época úmida. Confirmando este fato, as análises de termodinâmica (CAPE e CIN) foram menores do que os valores já observados para as estações seca e chuvosa.

Constatou-se que os cálculos de hi pelo software da REMTECH não representa a altura da CLN, mas uma estimativa da CR, apresentando diferença de 557,4 m entre as médias das alturas. Com o método de vento máximo proposto, a altura da CLN obteve uma média de 263,5 $\pm 121,7 \mathrm{~m}$, embora não consiga representar o surgimento da CLN nos horários de pôr-do-sol. A RS também registrou ocorrências de vento mais forte na altura da CLN, reforçando a teoria do vento máximo.
Embora usando técnicas de medidas diferentes, os equipamentos de medidas in situ (BC e RS) mostram uma concordância entre si melhor do que ao serem comparados com medidas remotas (SODAR), sendo que este último sempre superestima as medidas diretas.

Através das análises de regime turbulento foi possível encontrar uma predominância de regime de pouca estabilidade, abrangendo limites numéricos de pouca $(\varepsilon 1=20,5)$ e muita estabilidade $(\varepsilon 2=23,3)$, maiores do que no período chuvoso, indicando assim, fluxos mais elevados e maior tolerância a regimes pouco estáveis durante à noite.

\section{REFERÊNCIAS BIBLIOGRÁFICAS}

ACEVEDO O. C.; MORAES, O. L. L.; SILVA, R.; FITZJARRALD, D. R.; SAKAI, R. K.; STAEBLER, R. M.; CZIKOWSKY, M J. Inferring nocturnal surface fluxes from vertical profiles of scalars in an Amazon pasture. Global Change Biology, v. 10, n. 1-9, p. 886-894, 2004.

ARYA, S. P. In: Introduction to micrometeorology. 2nd ed., Academic Press, 415 p., 2001.

COSTA, M. H.; YANAGI, S. N. M. Effects of Amazon deforestation on the regional climate-Historical perspective, current and future research. Revista Brasileira de Meteorologia, v. 21, n. 3, p. 200-211, 2006.

FEARNSIDE, P. M.. Desmatamento na Amazônia: dinâmica, impactos e controle. Acta Amazônica, v. 36, n. 3, p. 395400, 2006. (doi: 10.1590/S0044-59672006000300018).

FISCH, G. F. Camada Limite Amazônica: aspectos observacionais e de modelagem. 1995. 202 f. (INPE-6123TDI/584). Tese (Doutorado em Meteorologia) - Instituto Nacional de Pesquisas Espaciais, São José dos Campos, SP, 1995. Disponível em: <http://urlib.net/sid.inpe.br/MTCm13@80/2005/08.30.17.33>

FISCH, G.; TOTA, J.; MACHADO, L. A. T.; SILVA DIAS, M. A. F.; LYRA, R. F. DA F.; NOBRE, C. A.; DOLMAN, A. J.; GASH, J. H. C. 2004. The convective boundary layer over pasture and forest in Amazonia. Theoretical and Applied 
Climatology, v. 78, n. 1-3, p. 47-59, 2004. (doi 10.1007/ s00704-004-0043-x).

GODOY, J. R. L.; MARABESI, M. A.; MORTARI, L. C.; AIDAR, M. P. M.; BUCKERIDGE, M. S. A dinâmica da floresta neotropical e as mudanças climáticas globais. Naturalia, v. 32, p. 53-66, 2009.

HOLTSlaG, A. A. M.; DUYNKERKE, G. G. Clear and Cloudy Boundary Layer. Royal Netherlands Academy of Arts and Sciences, Amsterdam, 371 p., 1998.

HOLTSLAG, A. A. M.; NIEUWSTADT, F. T. M. Scaling the atmospheric boundary layer. Boundary-Layer Meteorology, V. 36, N. 1-2, P. 201-209, 1986.

INPE. Projeto PRODES: monitoramento da floresta Amazônica Brasileira por satélite. Disponível em: $<$ http:// www.obt.inpe.br/prodes/ >. Acesso em: 14 Mar. 2009.

MAHRT, L.; SUN, J.; BLUMEN, W.; DELANY, T.; ONCLEY, S. Nocturnal boundary layer regimes. Boundary-Layer Meteorology, v. 88, n. 2, p. 255-278, 1998.

MOTA, M. A. S.; NOBRE, C. A. Relação da variabilidade da energia potencial convectiva disponível (CAPE) com a precipitação e a Alta da Bolívia durante a campanha "WETAMC/LBA". Revista Brasileira de Meteorologia, v. 21, n. 3b, p. 344-355, 2006.

OLIVEIRA, J. P.; FISCH, G. Efeito da turbulência na camada limite atmosférica em áreas de floresta e pastagem na Amazônia. Revista Brasileira de Meteorologia, v. 15, n. 2, p. 39-44, 2000.

OYAMA, M. D.; NOBRE, C. A. A new climate-vegetation equilibrium state for tropical South America. Geophysical Research Letter. v. 30, n. 23, p. 2199, 2003.

SAKAI, R. K.; FITZJARRALD, D. R.; MORAES, O. L. L.; STAEBLER, R. M.; ACEVEDO, O. C.; CZIKOWSKY, M. J.; SILVA, R.; BRAIT, E.; MIRANDA, V. Land-use change effects on local energy, water, and carbon balances in an Amazonian agricultural field. Global Change Biology, v. 10, n. 5, p. 895-907, 2004.
SANTOS, R. M. N. dos. Estudos da Camada Limite Noturna na Amazônia. 2005. 168 f. (INPE-14477-TDI/1158). Tese (Doutorado em Meteorologia) - Instituto Nacional de Pesquisas Espaciais, São José dos Campos, SP, 2005a. Disponível em: $<$ http://mtc-m18.sid.inpe.br/col/sid.inpe.br/ jeferson/2005/05.25.18.40/doc/publicacao.pdf $>$

SANTOS, L. Análise e caracterização da camada limite convectiva em área de pastagem, durante o período de transição entre a estação seca e chuvosa na Amazônia (experimento RaCCI-LBA/Rondônia). 2005. 118 f. (INPE14049-TDI/1064). Dissertação (Mestrado em Meteorologia) - Instituto Nacional de Pesquisas Espaciais, São José dos Campos, SP, 2005b. Disponível em: <http://mtc-m17.sid. inpe.br/col/sid.inpe.br/jeferson/2005/06.15.16.59/doc/ publicacao.pdf $>$

SANTOS, R. M. N.; FISCH, G.; DOLMAN, A. J.; WATERLOO, M. Modelagem da Camada Limite Noturna (CLN) durante a época úmida na Amazônia, sob diferentes condições de desenvolvimento. Revista Brasileira de Meteorologia, v. 22, n. 3, p. 387-407, 2007.

STULL, R. B. An introduction to boundary layer meteorology. Kluwer Academic Publishers, Dordrecht, 666 p., 1988.

TOMBROU, M.; FOUNDA, D.; BOUCOUVALA, D. Nocturnal boundary layer height prediction from surface routine meteorological data. Meteorology Atmospheric Physics. v. 68, n. $3-4$, p. $177-186,1998$

VON RANDOW, C.; MANZI, A. O.; KRUIJT, B.; OLIVEIRA, P. J.DE; ZANCHI, F. B.; SILVA, R. L.; HODNETT, M. G.;GASH, J. H. C.; ELBERS, J. A.; WATERLOO, M. J.; CARDOSO, F. L.; KABAT, P. Comparative measurements and seasonal variations in energy and carbon exchange over forest and pasture in South West Amazonia. Theoretical and Applied Climatology. v. 78, n. 1-3, p. 5-26, 2004. 